International Mathematical Forum, Vol. 9, 2014, no. 28, 1357 - 1361

HIKARI Ltd, www.m-hikari.com

http://dx.doi.org/10.12988/imf.2014.4227

\title{
A Fixed Point Theorem of Caccioppoli - Kannan Type on a Class of Generalized Metric Spaces
}

\author{
Hiranmay Dasgupta
}

JIS College of Engineering, Phase-III

Block-A, Kalyani, Nadia

Pin-741235, West Bengal, India

Sucharita Chakrabarti

Guru Nanak Institute of Technology

157/F Nilgunj Road, Panihati, Kolkata-700114

West Bengal, India

Prabir Chakraborty

Haldia Institute of Technology

ICARE Complex, HIT Campus, PO.-HIT, Haldia-721657

Purba Medinipur, West Bengal, India

Copyright (c) 2014 Hiranmay Dasgupta, Sucharita Chakrabarti and Prabir Chakraborty. This is an open access article distributed under the Creative Commons Attribution License, which permits unrestricted use, distribution, and reproduction in any medium, provided the original work is properly cited.

\begin{abstract}
In this paper we prove a Caccioppoli type Kannan's fixed point theorem in g.m.s. that includes Kannan's fixed point theorem in g.m.s. of P.K. Saha (Th. 2.1[4]) and of Dorel Mihet (Th. 2.1[5]).
\end{abstract}

Mathematics Subject Classification: Primary 54H25, Secondary 47H10

Keywords: Generalized metric space, T-orbitally complete, Fixed point 


\section{Introduction}

Recently Branciari [1] introduced the concept of a generalized metric space where the triangular inequality of a metric space has been replaced by a more general inequality involving four points instead of three.In this paper we generalized Caccioppoli-type fixed point theorem of Hiranmay Dasgupta[2] in generalized metric spaces (Th.3.1) and it is proved (Th.3.2) that the Kannan's fixed point theorem in generalized metric space([4],[5])follows from our theorem (Th.3.1).For our study we need the following known definitions and results.

\section{Preliminaries}

Definition 2.1 [1]. Let $X$ be a non-void set and $d: X \times X \rightarrow R^{+}$(nonnegative reals) be a mapping such that for $x, y \in X$ and for all distinct points $\xi, \eta \in X$, each of them different from $x$ and $y$, one has

$$
\begin{aligned}
d(x, y) & =0 \text { if and only if } x=y ; \\
d(x, y) & =d(y, x) ; \\
\text { and } \quad d(x, y) & \leq d(x, \xi)+d(\xi, \eta)+d(\eta, y) \quad \text { [rectangular property], }
\end{aligned}
$$

then $(X, d)$ is called a generalized metric space, (shortly a g.m.s.) and $d$ is called a generalized metric for $X$.

Definition 2.2 [1]. Let $(X, d)$ be a g.m.s. A sequence $\left\{x_{n}\right\}$ in $X$ is said to be a Cauchy sequence if for every $\epsilon>0$, there exists a positive integer $N$ such that $d\left(x_{n}, x_{n+m}\right)<\epsilon$ whenever $n \geq N$ and $m=1,2, \ldots$... g.m.s. $(X, d)$ is called complete if every Cauchy sequence in $X$ is convergent in $X$.

Definition 2.3 [1]. Let $T: X \rightarrow X$ be a mapping where $X$ is a g.m.s. For each $x \in X$, let $O(x, \infty)=\left\{x, T x, T^{2} x, T^{3} x, \cdots\right\}$. Then $X$ is said to be $T$ orbitally complete if and only if every Cauchy sequence which is contained in $O(x, \infty)$ for some $x \in X$ converges in $X$.

Remark 2.4 Every complete g.m.s. $X$ (where a mapping $T: X \rightarrow X$ is defined) is T-orbitally complete but a T-orbitally complete g.m.s. X need not be complete ([3], Note, p 590). 
Theorem 2.5 Caccioppoli type fixed point theorem[2]:Let $(X, \rho)$ be a complete metric space and let $T$ be a mapping of $X$ into itself. Suppose that for each positive integer $n, \rho\left(T^{n} x, T^{n} y\right) \leq a_{n}[\rho(x, T x)+\rho(y, T y)]$, for all $x, y \in X$ where $a_{n}(>0)$ is independent of $x, y$ and $0<a_{1}<1$. Than if the series $\sum_{i=1}^{\infty} a_{n}$ is convergent, $T$ has a unique fixed point in $X$.

\section{New Results}

Theorem 3.1 Let $(X, d)$ be a generalized metric space and $T: X \rightarrow X$ be a mapping such that $(A): d\left(T^{n} x, T^{n} y\right) \leq a_{n}[d(x, T x)+d(y, T y)]$ for all $x, y \in X$, $a_{n}(>0)$ is independent of $x, y$ and $0<a_{1}<\frac{1}{2}$. If $X$ is T-orbitally complete with respect to the generalized metric $d$, and $\sum_{i=1}^{\infty} a_{n}$ is convergent, then $T$ has a unique fixed point in $X$.

Proof. We note that $\mathrm{T}$ satisfies the following:

(B): $d\left(T^{n} x, T^{n+1} x\right) \leq\left(\frac{a_{1}}{1-a_{1}}\right)^{n} d(x, T x), x \in X, n \in N$ where $0<\frac{a_{1}}{1-a_{1}}<1$, which easily follows from (A) using method of induction. We also observe that if $\mathrm{T}$ has a fixed point it is unique by the condition (A) of the theorem.

We now prove the theorem considering the following two cases:

Case $\mathbf{P}$. In this case we assume that $T^{m} x \neq T^{n} x$ for $m, n \in N, m \neq n$ and $x \in X$. For $x, y \in X$, we define $\rho(x, y)= \begin{cases}d(x, T x)+d(y, T y), & \text { for } x \neq y \\ 0, & \text { for } x=y\end{cases}$

So for $x \neq y, \rho(x, y) \neq 0$ as $\mathrm{T}$ cannot have more than one fixed point. Again since $\rho(x, y)=d(x, T x)+d(y, T y) \leq d(x, T x)+2 d(z, T z)+d(y, T y)=$ $\rho(x, z)+\rho(z, y)$, for $x, y, z \in X$ with $x \neq y, \rho$ is a metric on X.In this metric space $(X, \rho)$ it is verified that $\mathrm{T}$ satisfies the results $(\mathrm{C})$ and $(\mathrm{D})$, where

(C): $\rho\left(T^{n} x, T^{n} y\right) \leq b_{n} \rho(x, y)$ for $n \in N, x, y \in X, b_{n}=\frac{a_{n}}{1-a_{1}} .(>0)$ and $b_{n}$ is independent of $x, y$, and

(D): $d\left(T^{n} x, T^{m} x\right) \leq 2 \rho\left(T^{n} x, T^{m} x\right)$ for any $x \epsilon X$ and $m, n \epsilon N, m \geq n$.

We now show that $\left\{T^{n} x\right\}$ is a $\rho$-Cauchy sequence in the metric space $(X, \rho)$.

For, if $m, n \in N$ with $m>n$, then $\rho\left(T^{n} x, T^{m} x\right) \leq \sum_{k=n}^{m-1} \rho\left(T^{k} x, T^{k+1} x\right)$

$=\sum_{k=n}^{m-1} \rho\left(T^{k} x, T^{k}(T x)\right) \leq \sum_{k=n}^{m-1} b_{k} \rho(x, T x)$, using $(\mathrm{C})$. As the series $\sum_{n=1}^{\infty} a_{n}$ is con-

vergent, $\sum_{n=1}^{\infty} b_{n}$ is also convergent. Let $\lambda$ be the positive integer such that $\lambda>\rho(x, T x)$. Then for an arbitrary $\epsilon(>0)$, there exists a positive integer p such that $\sum_{k=n}^{m-1} b_{k}<\frac{\epsilon}{\lambda}$ if $m>n \geq p$. Then for $m>n \geq p$, we have 
$\rho\left(T^{n} x, T^{m} x\right)<\frac{\epsilon}{\lambda} \rho(x, T x)<\epsilon$. Thus $\left\{T^{n} x\right\}$ is a $\rho$-Cauchy sequence in $(X, \rho)$ contained in $O(x, \infty)$.Hence from (D) it follows that $\left\{T^{n} x\right\}$ is also a d-Cauchy sequence in $(X, d)$ contained in $O(x, \infty)$. As $(X, d)$ is T-orbitally complete, $d\left(T^{n} x, u\right) \rightarrow 0$ as $n \rightarrow \infty$ for some $u \in X$. We now show that $T u=u$. We observe that $T^{n} x \neq u, T u$ for any $n \in N$; for if $T^{k} x=u$ or $T^{k} x=T u$, for some $k \in N, x \in X$, then it follows that $\left\{T^{n} u\right\}$ is a sequence with the following properties in $(X, d)$ :

$$
\begin{aligned}
& \text { (i) } \lim _{n \rightarrow \infty} T^{n} u=u, \quad(i i) T^{n} u \neq u \text { for any } n \in N \\
& \text { (iii) } T^{p} u \neq T^{r} u \text { for } p, r \in N, p \neq r .
\end{aligned}
$$

So $d\left(T^{n+1} u, T u\right) \leq a_{1}\left[d\left(T^{n} u, T^{n+1} u\right)+d(u, T u)\right] \leq a_{1}\left[\left(\frac{a_{1}}{1-a_{1}}\right)^{n} d(u, T u)+d(u, T u)\right]$, using (B) and letting $n \rightarrow \infty$, we get $d(u, T u) \leq a_{1} d(u, T u)$ which implies $T u=u$ as $a_{1}<1$, which contradicts (ii). Thus $T^{n} x \neq u, T u$ for any $n \in N$. Now using the rectangular inequality and (A), we obtain,

$$
\begin{aligned}
d(u, T u) \leq & d\left(u, T^{n} x\right)+d\left(T^{n} x, T^{n+1} x\right)+d\left(T^{n+1} x, T u\right) \\
\leq & d\left(u, T^{n} x\right)+d\left(T^{n} x, T^{n+1} x\right)+a_{1}\left[d\left(T^{n} x, T^{n+1} x\right)+d(u, T u)\right] . \\
\text { So, } d(u, T u) & \leq \frac{1}{1-a_{1}}\left[d\left(u, T^{n} x\right)+\left(1+a_{1}\right) d\left(T^{n} x, T^{n+1} x\right)\right] \\
& \leq \frac{1}{1-a_{1}}\left[d\left(u, T^{n} x\right)+\left(1+a_{1}\right)\left(\frac{a_{1}}{1-a_{1}}\right)^{n} d(x, T x)\right], \text { using (B), and letting } \\
n \rightarrow \infty & \text { we get } d(u, T u)=0, \text { i.e., } T u=u .
\end{aligned}
$$

Case Q. In this case we assume that $T^{m} x=T^{n} x$, for some $m, n \in N, m \neq n$. Let $m>n$, then $T^{m-n}\left(T^{n} x\right)=T^{n} x$,i.e., $T^{k} y=y$ where $k=m-n \geq 1, y=T^{n} x$. Now for $k=1, T y=y$ and for $k>1, d(y, T y)=d\left(T^{k} y, T^{k+1} y\right) \leq\left(\frac{a_{1}}{1-a_{1}}\right)^{k} d(y, T y)$, using (B) and so $T y=y$ as $0<\frac{a_{1}}{1-a_{1}}<1$.Thus from cases $\mathrm{P}$ and $\mathrm{Q}$ we see that $\mathrm{T}$ has a fixed point in $X$. This proves the theorem.

We now show that Kannan's fixed point theorem in generalized metric space follows from our Theorem-3.1.

Theorem 3.2 (Kannan's fixed point theorem in g.m.s. ([4], [5])). Let $(X, d)$ be a generalized metric space and $T$ be a mapping such that $T$ : $X \rightarrow X$ and $T$ satisfies the condition: $d(T x, T y) \leq \beta[d(x, T x)+d(y, T y)]$, where $0<\beta<1 / 2$ and $x, y \in X$. If $X$ is T-orbitally complete in $(X, d)$ then $T$ has a unique fixed point in $X$.

Proof. We have, $d(T x, T y) \leq \frac{\beta}{\alpha} \alpha[d(x, T x)+d(y, T y)]$, where $0<\alpha=\frac{\beta}{1-\beta}<1$.Again $d\left(T^{2} x, T^{2} y\right) \leq \beta\left[d\left(T x, T^{2} x\right)+d\left(T y, T^{2} y\right)\right]$. Now $d\left(T x, T^{2} x\right)=d(T x, T(T x)) \leq \beta\left[d(x, T x)+d\left(T x, T^{2} x\right)\right]$, which gives $d\left(T x, T^{2} x\right) \leq \frac{\beta}{1-\beta} d(x, T x)=\alpha d(x, T x)$.

Similarly $d\left(T y, T^{2} y\right) \leq \alpha d(y, T y)$.

Hence, $d\left(T^{2} x, T^{2} y\right) \leq \beta \alpha[d(x, T x)+d(y, T y)]=\left(\frac{\beta}{\alpha}\right) \alpha^{2}[d(x, T x)+d(y, T y)]$. 
Hence by induction, $d\left(T^{n} x, T^{n} y\right) \leq \frac{\beta}{\alpha} \alpha^{n}[d(x, T x)+d(y, T y)]$

i.e., $d\left(T^{n} x, T^{n} y\right) \leq a_{n}[d(x, T x)+d(y, T y)]$ where $a_{n}=\left(\frac{\beta}{\alpha}\right) \alpha^{n}>0$,

which is independent of $x, y \in X$ and $a_{1}=\frac{\beta}{\alpha} \alpha=\beta<\frac{1}{2}$. Now the series $\sum_{n=1}^{\infty} a_{n}$ is convergent. Hence by Theorem 3.1, T has a unique fixed point in $X$.

\section{References}

[1] A. Branciari, A fixed point theorem of Banach-Caccioppoli type on a class of generalized matric spaces, Publ. Math. Debrecen, (57) 1-2 (2000), pp $31-37$.

[2] Hiranmay Dasgupta, A Caccioppoli type fixed point theorem that includes Kannan's fixed point theorem, Proc. National Symposium, Math. Dept., Kalyani Univ. (West Bengal, India) 26-27 March (1999).

[3] B. K. Lahiri and Pratulananda Das, Fixed point of a LjubomirCiric's quasi - contraction mapping in a generalized matric space, Publ. Math. Debrecen, 61, 3-4(2002), pp 589-594.

[4] P. K. Saha and R. Tiwari, An alternative proof of Kannan's fixed point theorem in generalized matric spaces, News Bull. Cal. Math. Soc., 31, (4-6)(2008) 15-18.

[5] Dorel Mihet, On Kannan fixed point principle in generalized matric spaces, J. Nonlinear Sci. Appl. 2(2009), no.2, pp 92-96.

Received: February 11, 2014 